Megumi Wakabayashi

\title{
Schulromane von Robert Walser und Natsume Sōseki: Hybride Darstellungs- formen zwischen „hoch und niedrig“
}

\begin{abstract}
Robert Walser, a German-speaking Swiss author, wrote in Berlin a school novel Jakob von Gunten (1909), which is recognized to be a parody of a Bildungsroman. In this novel, Walser undermines the ideals of traditional education and dissolves the dualism between "domination and submission" through his narrative techniques. Around the same time, in Japan, Natsume Sōseki composed a school novel Botchan [The Fool from Tokyo] (1906), written in the style of entertainment literature. Behind the humorous style, Sōseki conceals his critical view of the radical modernization of Japan around the turn of the century. At a time of militaristic nationalism, both Walser in Europe and Sōseki in Japan criticized educational institutions as instruments for manipulation of public opinion in their novels, and succeeded in subverting the binary opposition between "high and low" literature.
\end{abstract}

Keywords: Robert Walser; Jakob von Gunten; Natsume Sōseki; Botchan; Schulroman

\section{Einleitung}

Um 1900 erschien eine ganze Reihe von Schulromanen, etwa von Hermann Hesse und Robert Musil, die die Qualen junger Schüler im repressiven Bildungssystem der bürgerlichen Gesellschaft thematisierten. Während hier jedoch klassische Bildung und moderne Wissenschaft auf dem Lehrplan stehen, lautet das Bildungsziel in Robert Walsers Roman Jakob von Gunten (1909) schlicht soziale Niedrigkeit. Schauplatz ist dabei sinnfälliger Weise eine Dienerschule. Jakob von Gunten ist somit unschwer als Parodie des Bildungsromans zu erkennen, mit dem Walser die Ideale traditioneller Bildung ironisch unterläuft. 
Zur gleichen Zeit schrieb Natsume Sōseki in Japan seinen Schulroman Botchan (Der Tor aus Tokio, 1906). ${ }^{1}$ Anders als in den deutschsprachigen Werken, die vor allem von den Agonien jugendlicher Helden erzählen, ist hier die Hauptfigur ein Lehrer. Mit ironischer Komik werden die Beziehungen der Kollegen untereinander und die täglichen Geschehnisse dargestellt. Im Vergleich zu seinen anderen Romanen ist Botchan eher im Ton trivialer Unterhaltungsliteratur verfasst, wobei in populärer Naivität das Gute gewinnt und das Böse unterliegt. Hinter der humorvollen Leichtigkeit verbirgt sich Sōsekis kulturkritischer Blick auf die radikale Modernisierung Japans um die Jahrhundertwende, unter der auch das Erziehungssystem einem tiefen Wandlungsprozess mit starken Verwerfungen ausgesetzt war. Gerade hierin überschreitet dieser Roman die Grenze der leichten Unterhaltung.

In diesem Beitrag geht es um die Frage, warum Schulromane gerade um 1900 sowohl im deutschsprachig-europäischen als auch im japanischen Kulturraum entstanden sind. Darüber hinaus soll gezeigt werden, auf welche Weise sie jenseits des Schemas „hoch und niedrig“ durch ihren hybriden Schreibstil neue Ausdrucksformen entwickeln.

\section{Robert Walsers Jakob von Gunten}

\subsection{Lehrer und Schüler}

Jakob von Gunten ist ein Roman in Tagebuchform, verfasst von dem gleichnamigen Protagonisten Jakob, der in die rätselhafte Dienerschule „Institut Benjamenta" eingetreten ist. Er schreibt über das Leben im Institut, über den Vorsteher Benjamenta, dessen Schwester, die Lehrerin Lisa, und über andere Lehrer und Mitschüler. Dazwischen erzählt er auch von seiner angeblich reichen Familie, vor allem seinem erfolgreichen Bruder. In der zweiten Hälfte des Romans wird allmählich klar, dass das Institut nicht mehr fortbestehen kann, dabei bleibt der Grund unerklärt. Die Zöglinge gehen einer nach dem anderen fort, Lisa verstirbt, zurück bleiben nur der Vorsteher und sein letzter Zögling Jakob. Am Ende verlassen auch die beiden gemeinsam das Institut und gehen in die Wüste.

1 Ein ausführlicher Vergleich in diese Richtung zwischen Walsers Geschwister Tanner und Sōsekis Ich der Kater findet sich bei Hintereder-Emde (2000). Namensschreibung in japanischer Weise mit dem Familiennamen vorangestellt. Sōseki ist ein Pseudonym, das in der Regel auch in der Forschung benutzt wird, der eigentliche Vorname lautet Kinnosuke. 
„Institut Benjamenta“ ist eine merkwürdige Schule, wie ihre Darstellung zeigt: Die Zöglinge sind hier „nur kleine, arme, abhängige, zu einem fortwährenden Gehorsam verpflichtete Zwerge“ (Walser 1985, 64). „(M)an lernt hier sehr wenig.“ (Walser 1985, 7). „Es fehlt eben [...] an Lehrkräften, das heißt die Herren Erzieher und Lehrer schlafen, oder sie sind tot, oder nur scheintot, oder sie sind versteinert, gleichviel, jedenfalls hat man gar nichts von ihnen.“ (Walser 1985, 9) Die Lehrer an diesem Institut bringen ihren Schülern keinerlei Kenntnisse bei und der Unterricht in diesem Institut hat mit Lehre und Erziehung, Bildung und Wissenschaft nichts zu tun. Die aufklärerische Idee einer modernen Bildung findet sich hier außer Kraft gesetzt. Die Zöglinge werden diszipliniert, um ein Nichts zu werden.

\subsection{Vorschrift und Disziplin}

Es gibt im Institut Benjamenta zahlreiche strenge Schulregeln: Die Zöglinge sollen sich z. B. beim Betreten und Verlassen des Vorsteherbüros in militärisch anmutender Form, „untertänig und höflich benehmen.“ (Walser 1985, 18) Sie stecken „in den eisernen Klauen der zahlreichen Vorschriften.“ (Walser 1985, 130) Diese bestimmen aber nicht nur ihr Benehmen, sondern auch jedes einzelne Körperorgan, wie folgende ins Grotesk-Sadistische getriebene Passage illustriert:

In der Unterrichtsstunde sitzen wir Schüler, starr vor uns herblickend, da, unbeweglich. Ich glaube, man darf sich nicht einmal die persönliche Nase putzen. Die Hände ruhen auf den Kniescheiben und sind während des Unterrichts unsichtbar. Hände sind die fünffingrigen Beweise der menschlichen Eitelkeit und Begehrlichkeit, daher bleiben sie unter dem Tisch hübsch verborgen. [...] Nasen von Zöglingen sollen stumpf und gestülpt erscheinen, so verlangen es die Vorschriften, die an alles denken, und in der Tat, unsere sämtlichen Riechwerkzeuge sind demütig und schamhaft gebogen. Sie sind wie von scharfen Messern kurzgehauen. Unsere Augen blicken stets ins gedankenvolle Leere, auch das will die Vorschrift. [...] Ziemlich ergötzlich sind die Ohren von uns Zöglingen. Sie wagen alle kaum zu horchen vor lauter gespannten Horchens. Sie zucken immer ein wenig, als fürchteten sie, von hinten plötzlich mahnend gezogen und in die Weite und Breite gerissen zu werden. [...] Schlägt der Ton eines Rufes oder Befehls an diese Ohren, so vibrieren und zittern die wie Harfen, die berührt und gestört worden sind. [...] Das Dressierteste an uns ist aber doch der Mund, er ist stets gehorsam und devot zugekniffen. [...] Lippen dürfen nicht prangen und lüstern blühen in der bequemen natürlichen Lage, sondern sie sollen gefalzt und gepreßt sein zum Zeichen energischer Entsagung und Erwartung. Das tun wir Schüler alle, wir gehen mit unsern Lippen laut bestehender Vorschrift sehr hart und grausam um, und daher sehen wir alle so grimmig wie kommandierende Wachtmeister aus. (Walser 1985, 55-56)

Zwar widersetzt sich Jakob dieser strengen Disziplin nicht offen, aber er ironisiert und demaskiert sie durch die Art seiner verniedlichenden Darstellung. Die 
Zöglinge sollen regelmäßig das Schulzimmer sowie Säbel und Helm über der Tür sorgfältig putzen. (Walser 1985, 35) Hier hängen auch die Bilder des verstorbenen Kaiserpaares, daher handelt es sich um eine Aufgabe, die dem Putzenden ein gewisses Maß an respekt- und würdevoller Haltung abverlangt. Jakob nennt aber gerade diese ernsthafte Aufgabe abschätzig eine „Zimmermädchenarbeit“ (Walser 1985, 35), denn jeder Zögling bindet sich dabei eine Schürze um. ${ }^{2}$ Ihr komisches Aussehen vergleicht Jakob mit den „märchenhaften Heinzelmännchen“ (Walser 1985, 36). Auf diese Weise wird die Atmosphäre der Erhabenheit des mit Kaiserbild und militärischen Insignien geschmückten Unterrichtsraums zerstört. Seine Beschreibung der Vorschriften und der Disziplin rückt diese ins Lächerliche und unterläuft auf diese Weise deren Unterdrückungscharakter.

\subsection{Herrschaft und Unterwerfung}

Jakob betont immer wieder den Kontrast zwischen dem riesigen Vorsteher und den winzigen Zöglingen. Er schreibt, „Herr Benjamenta ist ein Riese, und wir Zöglinge sind Zwerge gegen diesen Riesen“. (Walser 1985, 17) Benjamenta ist „allmächtig“ (Walser 1985, 140) und vor „solch ein[em] Herkules“ (Walser 1985, 18) sind Zöglinge nur „ein wenig gelbliche Blätter“ (Walser 1985, 140), die vom „Brausen und Zürnen“ (Walser 1985, 140) des Sturmwindes leicht weggeblasen werden. Es scheint, dass diese Beziehung zwischen Herrscher und Untergeordnetem unveränderbar ist, was aber keineswegs der Fall ist. Jakobs Haltung gegenüber dem Vorsteher wechselt zwischen Gehorsam und provozierender Frechheit, ja sogar Arroganz. Seine Klugheit nutzt er, um den Tyrannen bewußt in Rage zu bringen. „Seltsam, wieviel Lust es mir bereitet, Gewaltausübende zu Zornesausbrüchen zu reizen.“(Walser 1985, 44) Tatsächlich gelingt ihm dies immer wieder, um sich darüber heimlich lustig zu machen.

Wieder horchte ich draußen im Korridor am Schlüsselloch, und wieder blieb es ganz still. Ich strecke sogar ganz läppisch und echt zöglingshaft die Zunge heraus, und dann mußte ich lachen. Ich glaube, ich habe noch nie so gelacht. Natürlich ganz leise. Es war das denkbar echteste unterdrückte Gelächter. Wenn ich so lache, nun, dann steht nichts mehr über mir. Dann bin ich etwas an Umfassen und Beherrschen nicht zu Überbietendes. Ich bin in solchen Momenten einfach groß. (Walser 1985, 130)

Gerade Jakobs Gelächter ist es, das den Zorn des Schulleiters letztlich außer Kraft setzt, während sich der Zögling „einfach groß“ fühlt.

2 Claudia Liebrand (1999, 354-356) erläutert die Weiblichkeit der Zöglinge (gender trouble) und ihre Maskerade als Frauen. 
Schließlich erklärt der mächtige Vorsteher dem aufbegehrenden Zögling sogar seine Liebe. „Und noch ein drittes Geständnis: Ich habe eine seltsame, eine ganz eigentümliche, jetzt nicht mehr zu beherrschende Vorliebe für dich gewonnen." (Walser 1985, 94) Dabei fordert er Jakob nun seinerseits dazu auf, frech zu werden. Sein Zögling kommt dem nach, indem er seinen Herrscher „eisig kalt“ (Walser 1985, 106) behandelt. Hier schlägt die Beziehung zwischen Herrschaft und Gehorsam scheinbar gänzlich um. Aber nach wie vor nennt sich Benjamenta „dein Herr“ und den Schüler „meinen jungen armen Wurm, den ich, wenn's mich gelüstete, zermalmen könnte.“ (Walser 1985, 107) Er will seine Macht weiterhin zur Schau stellen und die Konstellation von Herrscher und Beherrschtem beibehalten. Dennoch geht von diesem „Wurm“ eine derart starke Wirkung aus, dass davon die Macht des „Riesen“ wirkungslos wird. Das Blatt, wie es im eingangs erwähnten Bild heißt, fällt zwar durch den Sturmwind sehr leicht ab, kann aber fröhlich und leicht in der Luft flattern und tanzen. Das Bild der hilflosen Schwäche kehrt sich nun ins Gegenteil einer freien, unbeschwerten Existenz. Und gerade dadurch gerät das gegensätzliche Schema „Herrschaft und Unterwerfung“ ins Taumeln. ${ }^{3}$

\section{Natsume Sōsekis Botchan (Der Tor aus Tokio)}

\subsection{Ein Kampf gegen die Ungerechtigkeit?}

Anders als bei Walser ist Sōsekis Protagonist „Botchan“ ${ }^{44}$ ein Lehrer. Nachdem er sein Studium in Tokyo absolviert hat, wird er Mathematiklehrer an einer ländlichen Mittelschule. Kurz nach seinem Antritt machen ihm die Schüler durch allerlei Streiche das Leben schwer. Er verlangt darum vom Konrektor, die Schüler gehörig zu bestrafen. Aber der Konrektor und andere Lehrer ignorieren seine Forderung. Nur sein Kollege „Stachelschwein“ will ihm als Einziger konsequent helfen und wird so ein Vertrauter des Neuankömmlings.

Der Konrektor und Yoshikawa, sein opportunistisch ergebener Verbündeter, handeln stets eigennützig und mit taktischen Hintergedanken. Der Konrektor raubt einem Englischlehrer sogar die Verlobte, während er diesen in eine abge-

3 Auf Jakobs performative Strategie des Zusammenbruchs von semantischen Oppositionen ist Liebrand (1999, 346-348) eingegangen. Dazu auch Hiebel 1999, 240-275.

4 „Botchan“ bezeichnet im Japanischen etwa Muttersöhnchen, einen ,grünen“ Jungen ohne Welterfahrung, auch einen verwöhnten Sohn reicher Eltern. Im Roman werden die meisten Figuren mit ähnlichen Spitznamen bezeichnet, wie im Folgenden „Stachelschwein“ (oder „Rothemd“). 
legene Gegend versetzt. Stachelschwein, der es gewagt hat, ihn in der Öffentlichkeit zu kritisieren, wird zum Rücktritt von seinem Lehrerposten gezwungen. Der Protagonist und sein Kollege Stachelschwein beschließen, diese „Laffen, Betrüger, Gauner, Heuchler, Schwindler, Bastarde, Spitzel und Burschen, die wie Hunde aussehen, wenn sie kläffen“ (Sōseki 2000, 88), ordentlich abzustrafen. Eines Abends verprügeln sie denn auch den Konrektor und seinen Kumpan, eine aus Botchans Sicht lang fällige und gerechte Strafe. Danach verlässt Botchan die Mittelschule und kehrt nach Tokyo zurück, um als Hilfsingenieur bei einer Straßenbahngesellschaft zu arbeiten.

Auf den ersten Blick handelt es sich um einen schemenhaft moralisierenden Roman, in dem der gute Held für die Gerechtigkeit kämpft und seine bösen Gegner niederringt. Diese Konstellation der typisierten Figuren entspricht zunächst ganz dem Muster eines vormodernen Trivialromans. Jedoch ist dieses Werk in der Tat nicht so einfach konzipiert.

\subsection{Wer gewinnt?}

Wer geht aus diesem Konflikt als Gewinner hervor? Ist es der Protagonist oder der Konrektor in seinem roten Flanellhemd? Der Konrektor wird fürs Erste gedemütigt, aber nicht öffentlich belangt, und verbleibt unverändert auf seinem Posten. Er gehört in der Mittelschule als einziger „Doktor der Philosophie“ (Sōseki 2000, 16) und Absolvent der neu geschaffenen Universität der Eliteschicht der geistigen Intelligenz an. Er repräsentiert das Karrieredenken der neuen Generation der Intellektuellen und weiß sich damit auf der gesellschaftlichen Gewinnerseite. Im Gegensatz dazu vertritt der Protagonist die traditionelle Moralität mit einem naiven Gerechtigkeitsempfinden. Einerseits kann sich der Protagonist als Vertreter der Gerechtigkeit moralisch überlegen fühlen, andererseits verlässt er die Schule, d.h. er gibt seine privilegierte Stellung als Lehrer auf. Damit nimmt er einen sozialen und auch wirtschaftlichen Abstieg in Kauf. Im Kontext der komplexen japanischen Modernisierung um die Jahrhundertwende, die den Hintergrund dieses Romans bildet, greift hier die simple Frage nach Gewinner und Verlierer nicht. Sie verdeutlicht vielmehr die Spannungen zwischen traditionellen und modernen Wertevorstellungen. 


\section{Reformen der Schulbildung in der japanischen Moderne}

\subsection{Reformen der Schulbildung}

Vor der Meiji-Restauration 1868, in der feudalistischen Edo-Zeit (1603-1868), gab es in allen Regionen des Landes Bildungseinrichtungen sowohl für den Kriegerstand (Hankō) als auch für das einfache Volk (Terakoya), in denen neben Konfuzianismus das Lesen, Schreiben und Rechnen unterrichtet wurden. Auch in der vormodernen Zeit bestand bereits ein über das ganze Land verbreitetes Bildungswesen, das eine sehr hohe Alphabetisierung der Bevölkerung bewirkte.

Nach der Restauration und Landeseröffnung hat die neue Regierung 1872 die allgemeine Schulpflicht eingeführt. „Das neue Grundgesetz zur Schulerziehung (Gakusei)“ orientierte sich am modernen westlichen Bildungswesen. Allerdings konnten die armen Schichten die Schulgebühren nicht bezahlen. Die Rate der Einschulung betrug darum nur 30 Prozent.

Infolgedessen wurde dieses liberale Erziehungsgesetz, das den aufklärerischen Bildungsidealen Frankreichs verpflichtet war, bereits 1879 wieder abgeschafft. 1880 wurde es durch das reaktionäre neue Erziehungsgesetz (KaiseiKyōikurei) ersetzt. Die Schulbildung wurde nun nach preußischem Vorbild stark vom Staat reglementiert.

Diese stark konservative Tendenz wurde noch durch das Kaiserliche Erziehungsedikt (Kyōiku-Chokugo) von 1890 verstärkt. Diese staatsideologische und nationalistische Ausrichtung wurde dabei durch den Konfuzianismus untermauert. Dadurch wurden Schülern und Lehrkräften eine extreme Vaterlandsliebe und Kaisertreue abverlangt. Der zentralistische Kaiserstaat plante die Modernisierung von oben her und instrumentalisierte das Erziehungswesen für die Stärkung von Staat und Militär, wie es Preußen und in seiner Folge das deutsche Kaiserreich so erfolgreich vorgeführt hatten. 1900 wurde das öffentliche Bildungssystem mit der Abschaffung der Unterrichtsgebühren vervollkommnet. 1905 lag die Einschulungsrate bei 95 Prozent.

\subsection{Sōseki und das Thema Schulbildung}

Sōseki (1867-1916) hat diesen Wandel der radikalen Modernisierung am eigenen Leibe erfahren: In der Grundschule erhielt er eine liberale Bildung, die aber ab der Mittelschule immer mehr von nationalistischer Ideologie dominiert wurde. 1890, im Jahr des Kaiserediktes, nahm er das Studium der Anglistik auf 
und nach dem Abschluss war er an verschiedenen Schulen als Englischlehrer tätig. 1895 trat er eine neue Stelle in Matsuyama an, wo sein Roman „Botchan“ spielt.

In diesem Roman werden die Lehrer, Angehörige der neuen Elite des modernen Schulsystems, die sich in ihrer privilegierten Überlegenheit zugleich auch für hervorragende Erzieher halten, schonungslos entlarvt: „Dann gibt es noch den Dachs, der immer nur zu sagen scheint: Wäre die Erziehung ein Lebewesen und zöge sich einen Gehrock an, dann sähe sie aus wie ich. - Jeder bildet sich auf seine Weise irgendetwas ein und nimmt sich furchtbar wichtig, [...]. " (Sōseki 2000, 68) Auch die Schüler werden kritisch dargestellt: „Die Schüler hatten sich nicht etwa entschuldigt, weil sie ehrlich bereuten, sondern weil es ihnen der Direktor befohlen hatte. Für sie war das Senken des Kopfes nur eine Formsache gewesen." (Sōseki 2000, 93) Die arrogante Unverbindlichkeit, die bereits auch die Schüler erfasst hat und nur dem Primat der Nützlichkeit verpflichtet ist, gibt den neuen Ton an. Deutlich zeichnet sich hier ab, dass dem Autor das modernisierte Bildungswesen in Japan suspekt war.

Japan hat 1895 den Ersten Japanisch-Chinesischen Krieg gewonnen und das ganze Land verfällt einer nationalistischen Stimmung. Sie wird im Roman in der Beschreibung einer Siegesfeier mit Feuerwerk festgehalten: „Aus einer der Raketen stieg ein Luftballon auf. Darauf stand: ,Es lebe das Kaiserreich!‘ [...] Von neuem stieg ein Ballon in die Höhe. Diesmal stand auf rotem Grund mit weißer Schrift: ,Es lebe Heer und Marine!““ (Sōseki 2000, 99)

Die zentralistische Meiji-Regierung in Japan hat auf Biegen und Brechen ohne die Basis einer aufgeklärten Bildung des Individuums eine jähe Europäisierung erzwungen. In dieser Zeit der oberflächlichen Modernisierung erlitt die Schulerziehung eine Phase der ethisch-moralischen Unterdrückung und Konfusion. Die Kluft zwischen einer rein technisch-institutionellen Modernisierung und einer gewachsenen Wertekultur wurde Sōseki nirgends stärker bewusst als während seines zweijährigen London-Aufenthalts.

\section{London-Erfahrung: Sōsekis Modernekritik}

1900 begab sich Sōseki für zwei Jahre nach London, um dort zu studieren. Er wurde vom japanischen Kulturministerium entsandt und sollte sich in Europas modernster Metropole die abendländische Wissenschaft $\mathrm{zu}$ eigen machen, um später zur Entwicklung der japanischen Wissenschaft beizutragen. Die Moderne, die er in London erlebte, hatte jedoch eine niederdrückende Wirkung auf ihn: Die Menschenmassen auf der Straße und der Lärm der Eisenbahnen brachten 
ihn nervlich an seine Grenzen. ${ }^{5}$ Das zivilisierte Leben in der großen Stadt fand er unmenschlich und hässlich. Er hatte schon damals eine dunkle Ahnung davon, dass der Fortschritt mit menschlicher Entfremdung und Naturzerstörung einhergehen werde. Seine Aufgabe, sich mit der europäischen Wissenschaft vertraut zu machen, schien ihm bald sinnlos zu sein, und so verlor er auch sein Ziel aus den Augen. Während in Japan die Verwestlichung und Modernisierung drastisch vorankamen, verstand er nun nicht mehr, worauf hin sich Japan entwickeln sollte. Nervlich schwer angeschlagen, kam er im Januar 1903 nach Japan zurück. Aber seine Zweifel und Orientierungslosigkeit teilten viele Intellektuelle Japans. Die japanische Modernisierung erwies sich für sie als ein geistiges Labyrinth.

\section{Berlin-Erfahrung: Walsers Ruhe in der Ruhelosigkeit}

Während Sōseki die Großstadt in verschiedenen kurzen Prosatexten konsequent negativ beschreibt, ${ }^{6}$ erscheint bei Walser die preußische Hauptstadt Berlin, in der er von 1905 bis 1913 lebte, in vitaler Lebendigkeit: „Noch nie, seit sie ist, hat in dieser Straße das Leben aufgehört zu leben. Hier ist das Herz, die unaufhörlich atmende Brust des großstädtischen Lebens.“ (Walser 1985/86, Bd. 3, 76) Diese großstädtische Lebendigkeit beschreibt Walser in seinen typischen Formulierungen des Entzückens:

\footnotetext{
Verpflichtet, verschuldet und verschwistert fühlt er sich dem sonderbaren Gerassel, Geräusche und Getöse. Das Hasten und Wehen empfindet er wie eine neblige, liebe Muttererscheinung. Er denkt nicht mehr daran, je wieder abzureisen. Mag es ihm gut oder schlecht gehen, mag er verkommen oder emporkommen, gleichviel, es ,hat' ihn, er ist für immer bezaubert, es ist ihm unmöglich, dieser großartigen Ruhelosigkeit Adieu zu sagen. (Walser 1985/86, Bd. 15, 51)
}

Anders als auf Sōseki wirken auf Walser der Lärm und das Dröhnen der Großstadt geradezu vertraut, und die Ruhelosigkeit selber strahlt für ihn eine paradoxe

5 Sōseki schreibt z. B. in der kurzen Geschichte Rondon Shōsoku (Nachrichten aus London, 1901), dass alle Fahrgäste in der Metro Zeitungen oder Zeitschriften lasen, während ihm von der Luft und dem Gerüttel so schlecht war, dass er Brechreiz verspürte. (Vgl. Natsume Sōseki Zenshū, Bd. 10, 656.)

6 Zum Beispiel in Rondon Shōsoku (Nachrichten aus London, 1901) und Rondon-To (London Tower, 1905). 
Ruhe aus. Wenn man sich ins Gedränge mischt, verschwindet „das Seelenübel“ (Walser 1985/86, Bd. 15, 55).

Im Tumult der Großstadt wird wie in einem chaotischen Schmelztopf aller Dualismus aufgelöst.

Arbeit und Vergnügen, Laster und guter Trieb, Streben und Müßiggang, Edelsinn und Niedertracht, Liebe und Hass, feuriges und höhnisches Wesen, Buntheit und Einfachheit, Armut und Reichtum schimmern, glitzern, blöden, träumen, eilen und stolpern hier wild und zugleich ohnmächtig durcheinander. (Walser 1985/86, Bd. 3, 76)

Auf diese Weise wird die Ruhelosigkeit in der großen Stadt zur ureigenen Ruhe, die divergenten Gegensätzlichkeiten werden amalgamiert.

\section{Schluss}

Um die Jahrhundertwende tendierten die Staaten Europas wie auch Japan zu einem stark militaristischen Nationalismus. Das Erziehungswesen wurde dabei zur weltanschaulichen Manipulation der Bevölkerung instrumentalisiert. Walser und Sōseki haben nahezu gleichzeitig in ihren Romanen die Bildungsinstitution Schule kritisch in den Blick genommen und bereits die Gefahren der Ideologisierung durch ihre jeweilige Darstellungsweise spürbar werden lassen.

Das Institut Benjamenta stellt eine Parodie der Ideale traditioneller Bildung dar, indem sein Bildungsziel die Niedrigkeit heißt. Es ist ein negativer Ausdruck des Bildungssystems der bürgerlichen Gesellschaft, in der man seiner immanenten Logik folgend eine höhere Stellung anstreben und aufsteigen muss. Aber Walser löst in seinem Anti-Bildungsroman dualistische Beziehungen wie „Herrschaft und Unterwerfung“ oder „groß und klein“ durch seine Sprachartistik auf und dabei verliert der Dualismus seine Gegensätzlichkeit. Auf diese Weise ist auch der Gegensatz „hoch und niedrig“ kein einfacher Widerspruch mehr. Bei Walser kann eine Höhe niedrig sein und eine Niedrigkeit hoch. Durch diesen paradoxen Wechsel stellt er den erstarrten Dualismus in Frage.

Sōsekis Botchan ist, auch wenn er auf den ersten Blick trivial-schematisch erscheint, kein leichter Unterhaltungsroman, worin zu guter Letzt das Gute über das Böse triumphiert. Hinter seiner naiven und unterhaltsamen Diktion versteckt sich eine ernsthafte Kritik an der japanischen Modernisierung. Sōseki, der sich im Dilemma zwischen der vormodernen, ethisch-moralischen Unterdrückung einerseits und der oberflächlichen Modernisierung andererseits sah und daraus keinen richtigen Ausweg finden konnte, schrieb einen Roman, der zwar unterhaltsam ist, aber keineswegs im Trivialen aufgeht. Bei Sōseki bleibt es strategisch unbestimmt, 
was trivial, was hochliterarisch sei, er bringt auf diese Weise das dominierende dualistische Schema von „hoch und niedrig“ ins Wanken und stellt zugleich die Frage nach den ethischen Werten in Zeiten der radikalen Modernisierung.

\section{Literaturverzeichnis}

\section{Werke von Natsume Sōseki}

Natsume Sōseki Zenshū (Sōseki Gesamtausgabe). 10 Bde. Tōkyō: Chikuma Shobō, 1987-1988. Der Tor aus Tokio. Aus dem Japanischen von Jürgen Berndt und Shinohara Seiei. Frankfurt/M.: Angkor, 2000.

\section{Werke von Robert Walser}

„Berlin und der Künstler“. Sämtliche Werke in Einzelausgaben. 20 Bde. Bd. 15. Hg. Jochen Greven. Zürich und Frankfurt/M.: Suhrkamp, 1985-1986. 49-51.

„Die Grossstadtstrasse“. Sämtliche Werke in Einzelausgaben. Bd. 15. Hg. Jochen Greven. Zürich und Frankfurt/M.: Suhrkamp, 1985-1986. 52-56.

„Friedrichstrasse“. Sämtliche Werke in Einzelausgaben. Bd. 3. Hg. Jochen Greven. Zürich und Frankfurt/M.: Suhrkamp, 1985-1986. 76-82.

„Jakob von Gunten“. Sämtliche Werke in Einzelausgaben. Bd 11. Hg. Jochen Greven. Zürich und Frankfurt/M.: Suhrkamp, 1985-1986.

\section{Sekundärliteratur}

Echte, Bernhard: „,Bedenkliches‘. Überlegungen zur Kulturkritik bei Robert Walser“. Robert Walsers, Ferne Nähe؛. Neue Beiträge zur Forschung. Hg. Wolfram Groddeck, Reto Sorg, Peter Utz und Karl Wagner. München: Wilhelm Fink, 2007. 203-275.

Hiebel, Hans H: „Robert Walsers Jakob von Gunten. Die Zerstörung der Signifikanz im modernen Roman“. Robert Walser. Hg. Klaus-Michael Hinz und Thomas Horst. Frankfurt/M., 1991. 240-275.

Hintereder-Emde, Franz: Ich Problematik um 1900 in der japanischen und deutschsprachigen Moderne. Studien zu Natsume Sōseki und Robert Walser. München: Indicium, 2000.

Liebrand, Claudia: „Jakob von Guntens Maskeraden. Spielkonfigurationen in Robert Walsers Tagebuchroman“. Colloquia Germanica 32.4 (1999): 345-362.

Yamazaki, Masakazu: Fukigen no Jidai. Yamazaki Masakazu Chosakushū (Die Zeit eines Mißbehagens. Gesammelte Schriften) Bd. 8. Tokyo: Chuuoukouron-Shinsha, 1981. 
Megumi Wakabayashi, Professorin für Germanistik an der Tokyo Gakugei Universität. Studium in Tokyo und Zürich. Forschungsschwerpunkte: Deutschsprachige Schweizer Literatur, besonders Robert Walser. Veröffentlichungen: Aufsätze über Robert Walser, Margrit Baur und die sprachliche Situation in der deutschsprachigen Schweiz. Übersetzungen ins Japanische von Texten Erika Burkarts, Ruth Schweikerts und Robert Walsers. 